\title{
Taking the plunge: Water immersion for labour and birth amongst women with moderate obstetric risk factors: A prospective cohort study
}

\author{
Emma Seed ${ }^{1}$, Lauren Kearney ${ }^{2}$, Edward Weaver ${ }^{1}$, and Rachael Nugent ${ }^{1}$ \\ ${ }^{1}$ Sunshine Coast University Hospital \\ ${ }^{2}$ Sunshine Coast Health Institute
}

August 28, 2021

\begin{abstract}
Objective: This study investigated maternal and fetal outcomes following warm water immersion (WWI) and/or waterbirth compared with land birth for women with moderate obstetric risk factors. Design: Prospective cohort study. Setting: Maternity hospital, Australia, 2019-2020 Population: 1665 participants, some with 'risk factors' for adverse perinatal outcomes requiring continuous electronic fetal monitoring (CEFM) during labour. Method: Multivariate logistical regressions were used to determine the odds of neonatal and maternal outcome measures between three groups: waterbirth, WWI and land birth Main outcome measures: Neonatal morbidity and mortality, including neonatal unit admission (NNU). Maternal clinical outcome measures, including mode of birth, perineal injury, postpartum haemorrhage, length of labour and morbidity. Results: NNU admissions for a suspected infectious condition were significantly higher in the land birth group ( $\mathrm{p}=0.035)$. After accounting for labour duration, epidural use, previous birth mode, and labour onset, no significant difference was detected between land births and WWI/water births in the odds of NNU admission $(\mathrm{p}=0.200)$. No babies were admitted to NNU with signs of water inhalation or drowning. Women birthing on land had a higher mean blood loss $(\mathrm{p}=0.036)$ and were more likely to be febrile ( $2 \%$ v $0 \% ; \mathrm{p}=0.007)$; Obstetric anal sphincter injury was similar between groups. Pharmacological analgesia use was lower in the WB/WWI group $(\mathrm{p}<0.001)$. There was 1 cord avulsion in the waterbirth group (0.41\%). Mode of birth was similar between groups $(\mathrm{p}=0.697)$. Conclusion: Despite moderate obstetric risk factors such as oxytocin administration and induction of labour; maternal and neonatal outcomes were similar between groups.
\end{abstract}

Title page

Full title

Taking the plunge: Water immersion for labour and birth amongst women with moderate obstetric risk factors: A prospective cohort study

\section{Running title}

Waterbirth in women with obstetric risk-factors

\section{Authors}

Dr Emma Seed ${ }^{1}$ (Corresponding author)

$+61439599489$

Emma.Seed@icloud.com

Dr Lauren Kearney ${ }^{2}$ 
$+61754565755$

Lkearney@usc.edu.au

Professor Ted Weaver ${ }^{1}$

Edward.Weaver@health.qld.gov.au

Dr Rachael Nugent ${ }^{1}$

Rachael.Nugent@health.qld.gov.au

${ }^{1}$ Department of Obstetrics and Gynaecology, Sunshine Coast Hospital and Health Service, QLD

${ }^{2}$ Sunshine Coast Health Institute, 6 Doherty St, Birtinya, QLD, 4575

Full title

Taking the plunge: Water immersion for labour and birth amongst women with moderate obstetric riskfactors: A prospective cohort study

Main Manuscript

\section{Introduction}

In 2018 the World Health Organisation ${ }^{(1)}$ published recommendations for intrapartum care, with an intentional focus on a positive childbirth experience as a significant end point for women undergoing labour and birth. Several studies suggest that warm water immersion (WWI) and waterbirth result in increased maternal satisfaction with the birth experience ${ }^{(2-4)}$, likely due to an increased sense of control ${ }^{(2,5,6)}$ and improved pain management ${ }^{(7)}$.

The practice is supported by several studies demonstrating reassuring clinical outcomes and increasing maternal preference for waterbirth ${ }^{(4)}$. Two recent systematic reviews ${ }^{(6,8)}$, demonstrate no increased risk of harm to the mother or neonate; rather evidence suggests afforded benefit including a reduction in the use of regional anaesthesia and length of labour, with no significant differences in neonatal morbidity ${ }^{(6,8)}$.

Whilst WWI has been both embraced and encouraged by midwives; obstetricians remain mostly reluctant to 'take the plunge' and support this practice, citing concerns over safety and a paucity of evidence. The American College of Obstetricians and Gynaecologists (ACOG) still recommends that birth occurs on land (9). Qualitative studies report a lack of support for WWI from obstetricians, most notably where women have additional risk factors $(10,11)$. This was particularly prevalent on obstetric units whereby senior staff did not appreciate the benefits of WWI and were seen to promote a medicalised approach to labour and birth (11). The Royal Australian and New Zealand College of Obstetricians and Gynaecologists (RANZCOG) acknowledges this lack of support for waterbirth amongst the medical community; suggesting that more research is required in the area ${ }^{(10)}$.

As a cohort, pregnant women are increasingly medically complex ${ }^{(12)}$, induction rates are rising ${ }^{(13)}$ and consequently the number of women requiring Continuous Electronic Fetal Monitoring (CEFM) is high. Whilst there is sound evidence to support WWI and waterbirth in low-risk women ${ }^{(6)}$, there is an absence of literature to support use in those with moderate obstetric risk-factors, who may be recommended to use CEFM during labour.

In our unit in Queensland, Australia, birthing pools were installed in 12 of 15 birth suites, and were equipped with wireless, waterproof CEFM, giving women unprecedented access to WWI and waterbirth. This study aims to investigate the maternal and fetal outcomes following WWI and/or waterbirth compared with land birth, including for those women with moderate obstetric risk factors, for example, those intending a vaginal birth after caesarean (VBAC) or requiring oxytocin administration.

\section{Methods}


We conducted a prospective cohort study of consecutive women planning a vaginal birth within the XXXXXXXXX Hospital, Queensland, Australia.

Participant sample and setting

A convenience sampling approach was taken. Within the Birthing Suite of the XXXXXXXXX Hospital, all women intending a vaginal birth and eligible to enter the birth pool (warm water immersion +/-water birth) between April 2019 through to April 2020 were included. The study site is a tertiary, publicly funded maternity unit with approximately 3600 births per year, in a coastal region of Queensland Australia.

Inclusion criteria (which were guided by the local Workplace Instruction on Warm Water Immersion and Water Birth ${ }^{(14)}$ ) were applied, including:

- Consent to CEFM where recommended (for example induction of labour)

- Cephalic presentation

- Term gestation

- Not a carrier of, or infected with, HIV, Hepatitis B or C

- $\mathrm{BMI}<35$

- Clear liquor (if membranes ruptured)

- No history of previous shoulder dystocia or severe postpartum haemorrhage

- No suspected fetal macrosomia (fetal weight $<4500 \mathrm{~g}$ )

Exclusion criteria:

- Emergency caesarean section for current birth

- Antepartum haemorrhage

- Pre-eclampsia

- Abnormal CTG prior to or at any time during the warm water immersion (Women were asked to leave the water, however, were still included in analysis as WWI only group )

The initial aim of this study was to compare the outcomes of women who birthed in water with those that had birthed on land. It is not possible to have a waterbirth and a caesarean section, hence women who underwent caesarean were excluded to ensure that we had homogeneity amongst the two groups. Following data collection, it became clear that we should evaluate labour outcomes amongst women who used WWI during labour but did not have a waterbirth. We decided that caesarean sections would continue to be excluded given that our primary area of interest was the outcomes of women achieving vaginal birth. In addition, according to the recent 2018 Cochrane Review, there is no evidence that WWI affects caesarean section rates ${ }^{(6)}$.

Groups

For analysis, groups were defined as follows:

Land birth: Laboured and birthed exclusively on land

Warm Water immersion : Laboured for all or part of the first stage of labour in water but birthed on land

Waterbirth: Laboured for all or part of the first stage of labour and remained in the water for birth

Water immersion for labour and/or birth workplace instruction

All women who entered the water were managed in accordance with our local WWI policy (appendix S1).

Data collection

Data was collected from two linked data sources: Birth Registration Short-form and the Queensland (Qld) State-wide Perinatal Data Collection. The primary outcome measure was admission to the NNU (see table 1 for full list of variables). 
[Insert table one near here]

All NNU admissions recorded on the perinatal database were manually cross checked against the electronic medical record. This confirmed that the data had been accurately recorded, thus enhancing internal reliability.

\section{Data analysis}

Descriptive analyses were performed to compare the maternal and fetal demographic and outcome variables for the three groups: land, water, and WWI-only births. For some analyses the water birth and WWI groups were combined into a single group (highlighted in the text below). Data are presented using median and inter-quartile range (IQR) for continuous data (due to skewed distributions), frequencies and proportions for categorical variables. Chi-square tests were performed to investigate associations between the groups (land, water, WWI) and categorical variables (e.g., NNU admission, perineal damage). Chi-square tests and Mann-Whitney U-tests were used to look for associations between NNU admission and maternal variables. Multivariate logistic regressions were used to model the odds of NNU admission, where variables were selected for inclusion based on their clinical relevance. These models contained the following variables: birth group (WWI vs waterbirth vs land birth; maternal age, maternal BMI, previous birth mode, epidural use, length of labour $\left(1^{\text {st }}+2^{\text {nd }}\right.$ stage) and labour onset. All analyses were performed in R (version 4.0.2).

Results

A total of 1665 women met inclusion criteria. There were 1265 land births, 243 water births and 157 WWI only land births. Baseline demographic and maternal delivery characteristics are presented in table 2 and were similar between groups.

[Insert table two near here]

Overall, the median length of WWI was 40 minutes (IQR: 15, 75). The median length of WWI for the WWI-only group (gave birth outside of water) was 45 minutes (IQR: 10, 72). The median length of WWI for the water birth group was 40 minutes (IQR: 15, 78.25).

Our primary outcome was admission to the neonatal unit (NNU). There was a significant association between the type of birth (land vs water/WWI) and NNU admissions ( $\mathrm{p}=0.022)$ whereby a higher proportion of land births were admitted to NNU compared to water/WWI births (11.2\% vs $7.3 \%)$. A sub-analysis, where instrumental births and those that had regional analgesia were removed, showed no significant association between the type of birth (land vs water/WWI) and NNU admissions ( $\mathrm{p}=0.774 ; 6.8 \%$ vs $6.3 \%$ for land vs water/WWI, respectively) occurred. No babies who underwent WWI/waterbirth required NICU admission for water inhalation or near drowning. There were no intrapartum deaths in any groups.

Differences were seen between groups for other outcome variables, with land births more likely to result in maternal pyrexia and suspected neonatal infection treated with prophylactic antibiotics. See table 3 for full summary.

[Insert table 3 near here]

Multivariate logistic regressions of neonatal admission (yes/no) adjusted for: maternal age, maternal BMI, previous birth mode, epidural use, total length of labour, and onset labour (in addition to birth group) were undertaken. After accounting for these variables, no significant difference was detected between land births and WWI/water births with respect to NNU admission $(\mathrm{p}=0.200)$ - see table 4.

[Insert table 4 near here]

There was a statistically significant association between the groups and admission to the NNU for a suspected infectious condition $(\mathrm{p}=0.035)$. $6.17 \%$ of land birth neonates were admitted to the NNU for this reason compared with $5.1 \%$ WWI and $2.06 \%$ in the waterbirth group. $5.45 \%$ of neonates born on land required the administration of antibiotics compared with $2.55 \%$ and $2.47 \%$ in the WWI/WB groups respectively. This had borderline significance $(\mathrm{p}=0.053)$. 
There was a significant difference between the groups in pharmacological analgesia use $(\mathrm{p}<0.001)$ where there was a much lower proportion of water births using pharmacological analgesia. In addition, significant differences were also observed between the groups in their first $(\mathrm{p}<0.001)$ and second $(\mathrm{p}<0.001)$ stage labour length where water births had the shortest median duration although this was not matched for parity. There was no significant difference between the proportion of SVB and instrumental births between land and WWI births $(\mathrm{p}=0.697)$. Waterbirths were not included here as they are unable to have an assisted birth.

When analysing rates of perineal trauma, we combined WWI/WB to form one group. Obstetric Anal Sphincter Injury was similar between groups (4.5\% of land births and $2.5 \%$ of water/WWI births had 3rd/4th degree lacerations). Overall, a similar proportion had no tears in each group (15.4\% land and $14 \%$ WWI/WB). A subgroup analysis was performed for those that did not have assisted deliveries (that is all spontaneous vaginal births), or epidurals, across all groups. A significant association between the type of birth (land vs water/WWI) and type of perineal trauma $(\mathrm{p}=0.021)$ was found in this group of patients. $7.7 \%$ of land births had an episiotomy and $5.5 \%$ of water/WWI births had episiotomies; $4 \%$ of land births and $2.6 \%$ of water/WWI births had 3rd/4th degree lacerations; slightly higher proportions of WWI/water births had 1st or 2nd degree tears.

There was a significantly higher proportion of patients with post-partum haemorrhage in the land birth group compared to the water/WWI birth group $(\mathrm{p}=0.036)$. There was 1 cord avulsion in the water birth group $(0.41 \%)$ with zero occurring in either the land or WWI groups.

\section{Discussion}

\section{Main results}

Our study found that babies born to women who utilised the birthing pool for WWI or waterbirth, despite some moderate risk factors, had lower rates of admission to the NNU and lower rates of observation for a suspected infectious condition and antibiotic administration. Encouragingly, this adds to existing evidence that WWI/Waterbirth is not associated with increased rates of NNU admission in low-risk women ${ }^{(6,8)}$. No babies required NNU admission for water inhalation or drowning.

The risk of neonatal sepsis is often cited by those who oppose WWI/Waterbirth ${ }^{(15)}$. However, case reports of neonates infected with $L$. pneumophila and $P$. aeruginosa ${ }^{(16)}$, appear to have resulted from a deviation from established waterbirth protocols and the use of unclean water. Overwhelmingly, recent literature suggests no increase in infective sequalae from WWI/WB, which is consistent with our findings ${ }^{(17,18)}$.

Most women (95.5\%) who were febrile intrapartum had an epidural, likely explaining the favourable results for the WWI/WB group. This association between maternal pyrexia and epidurals is well established with one retrospective study of 261,457 women finding those with an epidural five times more likely to experience pyrexia and neonates 1.6 times more likely to receive antibiotics ${ }^{(19)}$. The multivariate regression analysis removed women with epidurals from the cohort and subsequently showed no differences in NNU admission between the groups.

The role of waterbirth as a contributor to rates of perineal trauma is controversial. Our study showed similar rates of OASIS between land birth and WWI/WB. However, the subgroup analysis comparing spontaneous vaginal birth in women on land and in water, showed a significant association between land birth and increased rates of episiotomy. This land birth group did not include women who used WWI during labour and then birthed on land. A Cochrane review ${ }^{(6)}$, and several recent studies ${ }^{(20)}$, support the notion that WWI/WB does not increase rates of perineal trauma and our findings are consistent with this.

Our study suggested lower rates of PPH in the WWI/Waterbirth group, however estimating blood loss is notoriously inaccurate ${ }^{(21)}$, and more challenging in water. The waterbirth protocol within the study site recommends placental delivery occurs on land, thereby allowing blood loss to be more accurately estimated. The authors recognise that 'pool exit' is not always immediate and hence estimations of blood loss in the waterbirth group are more likely to be imprecise. Neiman ${ }^{(18)}$ suggests a small increase in PPH with 
waterbirth, but this is inconsistent with the broader literature $(6,20,22)$, which concur no clinical difference between groups.

Cord avulsion occurs when rapid cord traction overstretches umbilical cords that cannot reach above the water surface ${ }^{(23)}$. This is a rare event and studies are inevitably underpowered to prove a statistically significant association with waterbirth. According to Schafer ${ }^{23)}$, based on pooled data of 10,000 waterbirths, the incidence of cord avulsion appears increased at approximately 3.10 per 1000 . However, this risk can be mitigated by clear waterbirth protocols, midwifery education and discussion with women before pool entry $(20,23)$.

\section{Strengths and limitations}

This is the first study of its kind to include women utilising WWI and waterbirth whilst using CEFM. These women represent a 'moderate risk' rather than the usual 'low risk' cohort that has dominated the literature until now. The safety and efficacy of WWI for labour and birth in low-risk women is well established ${ }^{(6,8)}$. This study adds to the current literature but also highlights that this practice is a safe option for some women with clearly defined, moderate risk factors who require CEFM in labour.

This is a large sample size relative to other studies, with 400 women using WWI during their labour and/or birth. The groups had similar baseline characteristics and the subgroup analysis allowed for the control of confounding factors. Our data was prospectively collected and inputted into the perinatal database at the time of birth thereby increasing the reliability of our findings.

Due to the nature of the intervention, patients self-select Waterbirth/WWI which may be a source of bias. A randomised controlled trial would reduce bias but would be challenging given it removes choice around pool access and birth setting. Despite the larger sample size, some secondary outcomes are relatively rare (i.e. cord avulsion), and the nonsignificant findings should be interpreted within the context of limited statistical power.

Our department has a clear, well-defined policy surrounding WWI/Waterbirth and an established midwifery education programme facilitating training of accoucheurs in waterbirth. The findings from this study may not be generalisable to water immersion protocols at sites whereby the policies and education surrounding WWI/Waterbirth are less established or differ significantly from our study site.

\section{Conclusion}

There appears an increasing dichotomy between obstetricians and the women that we care for, with consumer perceptions that the former are keen to "over medicalise' both labour and birth ${ }^{(11)}$. The "medicalisation" of birth has diminished women's satisfaction with their experience ${ }^{(24)}$, and in recent times, there has been a substantial increase in the application of a range of practices to initiate, accelerate, regulate and monitor the physiological process of labour, with the aim of improving outcomes for women and babies ${ }^{(1)}$. Certainly, caesarean section and induction rates continue to rise ${ }^{(25)}$ without a concomitant reduction in perinatal mortality and morbidity, perhaps because women under hospital care have increasing numbers of medical comorbidities $^{(12)}$. Maternal request for caesarean and induction are also rising ${ }^{(25)}$ and obstetricians must tread a careful line between over and under intervention.

Despite these challenges, it is of paramount importance to provide safe, quality maternity care that is evidence-based, supports women, respects their choices for intrapartum care and promotes labour and spontaneous vaginal birth as normal, yet empowering life events. This is likely to be achieved by partnering more effectively with women and the maternity care team, ensuring that each person has a shared understanding of what the woman wants, and how that might be achieved under an umbrella of safe care.

Despite increasing numbers of women wanting access to and using WWI, peak bodies internationally are not supportive of waterbirth. The RCOG has no current guideline and NICE recommends that women should be offered WWI for pain relief but states that there is inadequate evidence to support or discourage waterbirth 
(26). RANZCOG cites data suggesting that waterbirth can be achieved safely but falls short of endorsing the practice ${ }^{(10)}$ and ACOG recommends that birth occur on land ${ }^{(9)}$.

The growing body of literature around WWI/Waterbirth suggests that hesitation and lack of support for waterbirth amongst obstetric staff is unfounded and not based on current evidence. Demand for access to non-pharmacological analgesia continues to increase and as a profession we need to ensure that we are flexible and proactive in meeting these demands. We must continue to work with, and listen to, pregnant women under our care and base recommendations and guidelines on current evidence, not personal bias. Where possible we should 'take the plunge' and support increasing access to water immersion, including for those women who have moderate obstetric risk factors. This must coincide with the development of evidence-based guidelines, audit of practice and the provision of suitable education to ensure that the practices of WWI and waterbirth are implemented safely.

\section{Disclosure of interests}

Nothing to declare

\section{Contribution to authorship}

Above order of authorship determined by relative contribution to the work, with RN being the most senior researcher and hence listed last.

- ES and EW study concept and research proposal

- ES, LK, RN study design

- ES, LK acquisition of data

- ER and LK data analysis

- ES, LK, RN interpretation of data

- ES draft article

- ES, LK, RN and EW critical revision and final approval

\section{Details of ethical approval}

This study was granted ethical approval through The Prince Charles Hospital Human Research Ethics Committee (HREC/17/QPCH/480). All data were deidentified prior to export and analysis. Individual consent was not sought as approval was granted through the Public Health Act (RD007551) to access routinely collected data. The study was conducted in accordance with the ethical principles from the National Health and Medical Research Council guidelines ${ }^{(27)}$.

\section{Funding}

None

\section{Acknowledgements}

The authors would like to acknowledge the Midwives and Doctors at the study site for their continued efforts to provide high quality, women centered care. We are grateful to Elizabeth Ryan at the Centre for Health Services Research, The University of Queensland, Brisbane for her expert assistance with statistical analysis and to Ada Moore, RM, for her data collection efforts.

\section{References}

1. World Health Organisation. WHO Recommendations: Intrapartum care for a postiive childbirth experience Geneva: World Health Organisation; 2018. Contract No.: Licence: CC BY-NC-SA 3.0 IGO.

2. Hall SM, Holloway IM. Staying in control: Women's experiences of labour in water. Midwifery. 1998;14(1):30-6.

3. Maude RM, Foureur MJ. It's beyond water: stories of women's experience of using water for labour and birth. Women \& Birth. 2007;20(1):17-24. 
4. Carlsson T, Ulfsdottir H. Waterbirth in low-risk pregnancy: An exploration of women's experiences. Journal of Advanced Nursing. 2020;76(5):1221-31.

5. Richmond H. Women's experience of waterbirth. The Practising Midwife. 2003;1(3):26-31.

6. Cluett ER, Burns E, Cuthbert A. Immersion in water during labour and birth. Cochrane Database of Systematic Reviews. 2018;2018(5).

7. Lopes F, Tereso A, Alice Curado M, Bértolo H, Carvalhal L, Guterres R. Hydrotherapy in the 1st stage of labor and its pain relief effects: a scoping review. Annals of medicine (Helsinki). 2019;51(sup1):207-.

8. Davies R, Davis D, Pearce M, Wong N. The effect of waterbirth on neonatal mortality and morbidity: a systematic review and meta-analysis. JBI database of systematic reviews and implementation reports. 2015;13(10):180-231.

9. American College of Obstetricians and Gynecologists. Committee Opinion: Immersion in water during labour and delivery. No. 679. Obstetrics and Gynecology. 2016;128:e231-6.

10. The Royal Australian and New Zealand College of Obstetricians and Gynaecologists. Water Immersion During Labour and Birth Melbourne: RANZCOG; 2021.

11. Milosevic S, Channon S, Hunter B, Nolan M, Hughes J, Barlow C, et al. Factors influencing the use of birth pools in the United Kingdom: Perspectives of women, midwives and medical staff. Midwifery. 2019;79.

12. Narayan B, Nelson-Piercy C. Medical problems in pregnancy. Clinical Medicine. 2017;17(3):251.

13. Walker S, van Rijn B, Macklon N, Howe D. The rising rate of labour induction: what is causing the trend? Archives of Disease in Childhood - Fetal and Neonatal Edition. 2014;99.

14. Women and Children's Service Group. Warm Water Immersion and Waterbirth: Workplace instruction. 2020 .

15. Harper B. Birth, bath, and beyond: the science and safety of water immersion during labor and birth. Journal of Perinatal Education. 2014;23(3):124-34.

16. Vanderlaan J, Hall P. Systematic Review of Case Reports of Poor Neonatal Outcomes With Water Immersion During Labor and Birth. The Journal of perinatal \& neonatal nursing. 2020;34(4):311-23.

17. Henderson J, Burns EE, Regalia AL, Casarico G, Boulton MG, Smith LA. Labouring women who used a birthing pool in obsteric units in Italy: prospective observational study. BMC Pregnancy and Childbirth. 2014;14(1):17.

18. Neiman E, Austin E, Tan A, Anderson CM, Chipps E. Outcomes of Waterbirth in a US Hospital-Based Midwifery Practice: A Retrospective Cohort Study of Water Immersion During Labor and Birth. Journal of Midwifery and Women's Health. 2020;65(2):216-23.

19. White A, Olson D, Messacar K. A state-wide assessment of the association between epidural analgesia, maternal fever and neonatal antibiotics in Colorado, 2007-2012. Archives of disease in childhood Fetal and neonatal edition. 2017;102(2):F120-F5.

20. Sidebottom AC, Vacquier M, Simon K, Wunderlich W, Fontaine P, Dahlgren-Roemmich D, et al. Maternal and Neonatal Outcomes in Hospital-Based Deliveries With Water Immersion. Obstetrics and gynecology. 2020;136(4):707-15.

21. Buckland SS, Homer CSE. Estimating blood loss after birth: Using simulated clinical examples. Women and Birth. 2007;20(2):85-8.

22. Burns EE, Boulton MG, Cluett E, Cornelius VR, Smith LA. Characteristics, interventions, and outcomes of women who used a birthing pool: a prospective observational study. Birth. 2012;39(3):192-202. 
23. Schafer R. Umbilical Cord Avulsion in Waterbirth. Journal of Midwifery \& Women's Health. 2014;59(1):91-4.

24. de Costa C, Robson S. Throwing out the baby with the spa water? Medical Journal of Australia. 2004;181(8):438-40.

25. BetráN AP, Temmerman M, Kingdon C, Mohiddin A, Opiyo N, Torloni MR, et al. Interventions to reduce unnecessary caesarean sections in healthy women and babies. The Lancet. 2018;392(10155):1358-68.

26. NICE (National Institute for Health and Clinical Excellence). Intrapartum care: care of healthy women and their babies durig childbirth. London2007.

27. National Health and Medical Research Council. National Statement on Ethical Conduct in Human Research - May, 2015 Update 2007 [Available from: https://www.nhmrc.gov.au/guidelines-publications/e72.

Tables and Figures

Table 1. Data collection

Maternal

Neonatal
Age (years) Body Mass Index (BMI) Parity (nulliparous / multiparous) Any Smoking during pregnancy Previous pregnancy (n) Mode of previous birth Previous caesarean section (n) Onset of labour (spontaneous or induced) CTG in labour (yes / no) FSE in labour (yes / no) Scalp $\mathrm{pH}$ (if collected and result if so) Lactate (if collected and result if so) Presentation at birth Labour and birth complications (intrapartum haemorrhage; postpartum haemorrhage; maternal pyrexia) Intention to birth in water (yes/no) Perineal damage (including tears and episiotomy) Pharmacological analgesia Duration of first and second stages of labour (minutes) Mode of birth Estimated blood loss (mls) Use of warm water immersion during labour (yes/no) Duration of warm water immersion (minutes)

Gestation at birth APGAR scores at 1 and 5 minutes Admission to Neonatal Unit (NNU) Neonatal sepsis Observation for potential risk of sepsis (not specified as neonatal sepsis) Observation for risk of sepsis if prophylactic antibiotics are commenced for $>=24$ hours

Table 2: Cohort characteristics

\begin{tabular}{lllll}
\hline & & & \multicolumn{2}{l}{ Warm water } \\
Variable & $\begin{array}{l}\text { Land birth } \\
(\mathrm{n}=1265) \mathrm{n}(\%)\end{array}$ & $\begin{array}{l}\text { immersion }(\mathrm{n}=157) \\
(\mathrm{n}=243) \mathrm{n}(\%)\end{array}$ & $\mathrm{n}(\%)$ & Total \\
\hline Maternal Age & $31(27,35)$ & $31(28,35)$ & $31(27,34)$ & $31(27,35)$ \\
Maternal BMI & $24(21.7,28)$ & $23(21,26)$ & $23.5(21.9,26)$ & $24(21.3,27)$
\end{tabular}




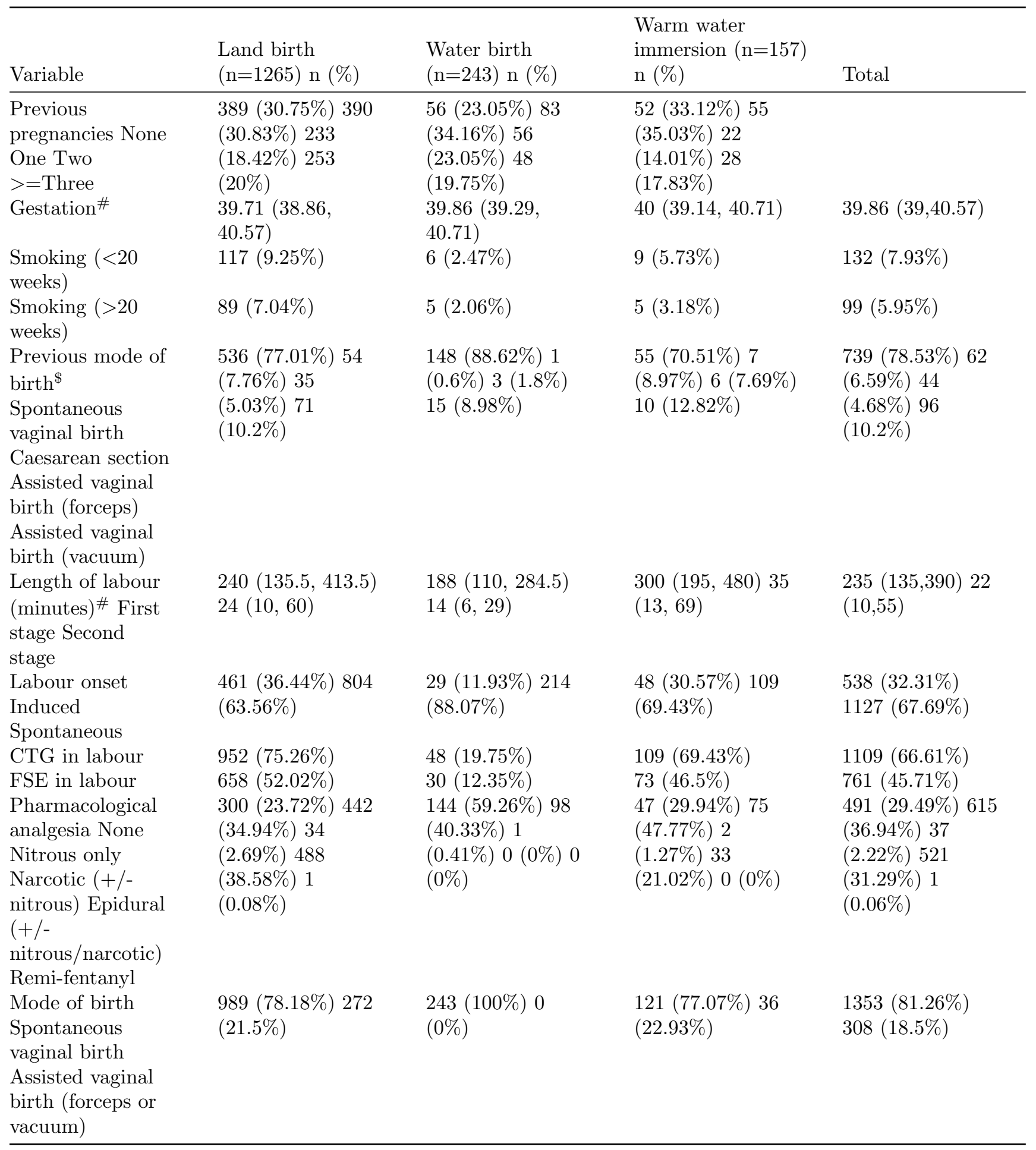

\# Median (IQR) ${ }^{\$}$ Multiparous only

Table 3. Categorical outcome variables 


\begin{tabular}{|c|c|c|c|c|}
\hline Variable & $\begin{array}{l}\text { Land birth } \\
(\mathrm{n}=1265) \mathrm{n}(\%)\end{array}$ & $\begin{array}{l}\text { Water birth } \\
(\mathrm{n}=243) \mathrm{n}(\%)\end{array}$ & $\begin{array}{l}\text { Warm water } \\
\text { immersion }(\mathrm{n}=157) \\
\mathrm{n}(\%)\end{array}$ & Total \\
\hline $\begin{array}{l}\text { Intrapartum } \\
\text { haemorrhage }\end{array}$ & $4(0.32 \%)$ & $0(0 \%)$ & $0(0 \%)$ & $4(0.24 \%)$ \\
\hline Primary PPH & $106(8.38 \%)$ & $13(5.35 \%)$ & $15(9.55 \%)$ & $134(8.05 \%)$ \\
\hline Maternal pyrexia & $22(1.74 \%)$ & $0(0 \%)$ & $0(0 \%)$ & $22(1.32 \%)$ \\
\hline $\begin{array}{l}\text { Neonatal } \\
\text { Morbidity }\end{array}$ & $839(66.32 \%)$ & $200(82.3 \%)$ & $102(64.97 \%)$ & $1141(68.53 \%)$ \\
\hline NNU admission & $142(11.23 \%)$ & $13(5.35 \%)$ & $16(10.19 \%)$ & $171(10.27 \%)$ \\
\hline $\begin{array}{l}\text { Observation of } \\
\text { neonate for } \\
\text { suspected } \\
\text { infectious } \\
\text { condition }\end{array}$ & $78(6.17 \%)$ & $5(2.06 \%)$ & $8(5.1 \%)$ & $91(5.5 \%)$ \\
\hline $\begin{array}{l}\text { Prophylactic } \\
\text { pharmacotherapy }\end{array}$ & $69(5.45 \%)$ & $6(2.47 \%)$ & $4(2.55 \%)$ & $79(4.7 \%)$ \\
\hline $\begin{array}{l}\text { Any perineal } \\
\text { damage }\end{array}$ & $1070(84.58 \%)$ & $206(84.77 \%)$ & $138(87.9 \%)$ & $1414(84.92 \%)$ \\
\hline $\begin{array}{l}\text { Severe perineal } \\
\text { damage }\left(3^{\text {rd }} / 4^{\text {th }}\right. \\
\text { degree })\end{array}$ & $57(4.51 \%)$ & $7(2.88 \%)$ & $3(1.91 \%)$ & $67(4.02 \%)$ \\
\hline $\begin{array}{l}\text { Episiotomy } \\
\text { Estimated Blood } \\
\text { Loss }\end{array}$ & $285(22.53 \%)$ & $0(0 \%)$ & $47(29.94 \%)$ & $332(19.94 \%)$ \\
\hline $0-499 \mathrm{ml}$ & $1022(80.79 \%)$ & $213(87.65 \%)$ & $130(82.8 \%)$ & $1365(81.98 \%)$ \\
\hline $500-999 \mathrm{ml}$ & $156(12.33 \%)$ & $23(9.47 \%)$ & $19(12.1 \%)$ & $198(11.89 \%)$ \\
\hline $1000-1499 \mathrm{ml}$ & $57(4.51 \%)$ & $4(1.65 \%)$ & $5(3.18 \%)$ & $66(3.96 \%)$ \\
\hline $1500-1999 \mathrm{ml}$ & $17(1.34 \%)$ & $2(0.82 \%)$ & $1(0.64 \%)$ & $20(1.2 \%)$ \\
\hline$>2000 \mathrm{ml}$ & $13(1.03 \%)$ & $1(0.41 \%)$ & $2(1.27 \%)$ & $16(0.96 \%)$ \\
\hline
\end{tabular}

Table 4. Multivariate regressions of neonatal admission.

\begin{tabular}{|c|c|c|c|c|c|}
\hline Parameter $^{a}$ & $\begin{array}{l}\text { NNU } \\
\text { Admission } \\
(\mathrm{N}=171)\end{array}$ & $\begin{array}{l}\text { No NNU } \\
\text { admission } \\
(\mathrm{N}=1494)\end{array}$ & $\mathrm{p}$-value ${ }^{\mathrm{b}}$ & OR $(95 \% \mathrm{CI})^{\mathrm{c}}$ & p-value ${ }^{c}$ \\
\hline \multicolumn{6}{|l|}{ Group } \\
\hline Land birth & $142(83.04 \%)$ & $1123(75.17 \%)$ & 0.022 & -ref- & - \\
\hline $\begin{array}{l}\text { Water } \\
\text { birth/WWI }\end{array}$ & $29(16.96 \%)$ & $371(24.83 \%)$ & & $0.70(0.40,1.18)$ & 0.200 \\
\hline Epidural & $89(52.05 \%)$ & $432(28.92 \%)$ & $<0.001$ & $\begin{array}{l}1.58(1.02, \\
2.44)\end{array}$ & 0.042 \\
\hline $\begin{array}{l}\text { Previous Birt } \\
\text { Mode }\end{array}$ & & & & & \\
\hline $\begin{array}{l}\text { No previous } \\
\text { birth/ } \\
\text { Caesarean } \\
\text { section }\end{array}$ & $114(66.67 \%)$ & $671(44.94 \%)$ & $<0.001$ & -ref- & - \\
\hline
\end{tabular}




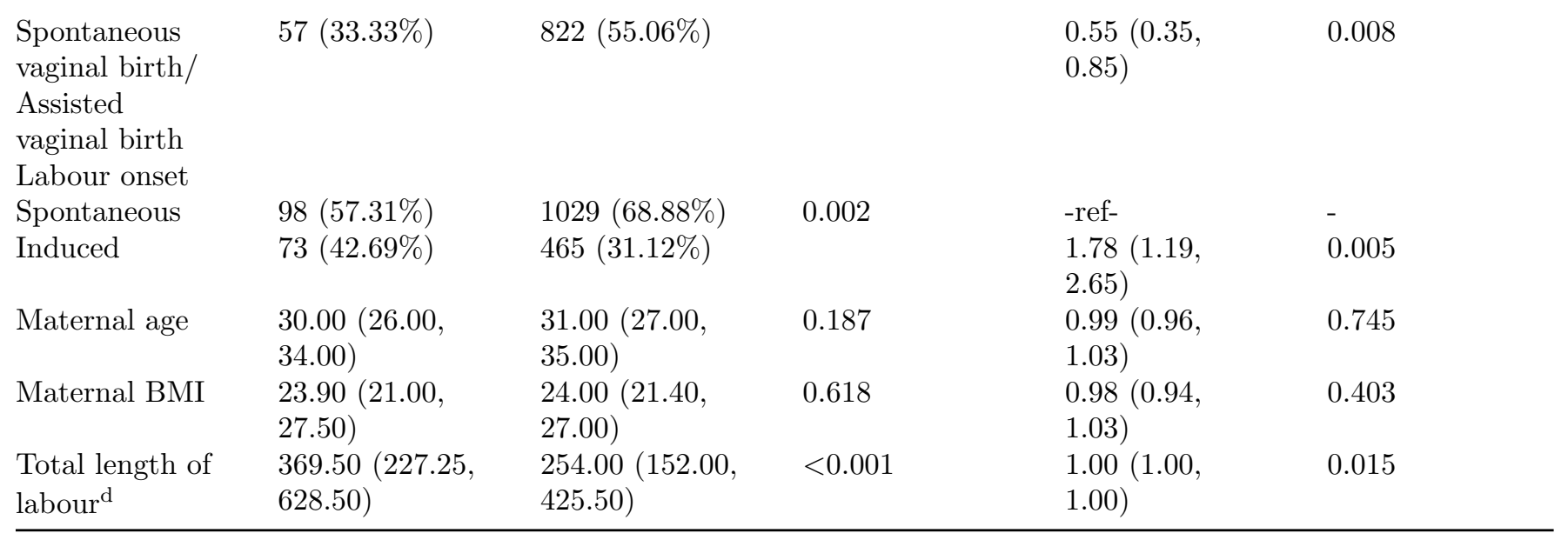

${ }^{\text {a }}$ Data are summarised as N (\%) or median (IQR) ${ }^{\mathrm{b}}$ Pearson's Chi-square test; Mann Whitney U test ${ }^{\mathrm{c}}$ Obtained from a multivariate logistic regression of neonatal admission containing all of the variables in the table above $^{\mathrm{d}} 1^{\text {st }}+2^{\text {nd }}$ stage length (min) 